\title{
The combination strategies will be ready the right first-line choice for squamous lung cancer patients?
}

\author{
Cesare Gridelli, Francesca Casaluce \\ Division of Medical Oncology, S.G. Moscati Hospital, Avellino, Italy \\ Correspondence to: Cesare Gridelli. Division of Medical Oncology, S.G. Moscati Hospital, Avellino, Italy. Email: cgridelli@libero.it. \\ Comment on: Paz-Ares L, Luft A, Vicente D, et al. Pembrolizumab plus chemotherapy for squamous non-small cell lung cancer. N Engl J Med 2018. \\ [Epub ahead of print].
}

Submitted Nov 14, 2018. Accepted for publication Nov 19, 2018.

doi: $10.21037 /$ tlcr.2018.11.02

View this article at: http://dx.doi.org/10.21037/tlcr.2018.11.02

Squamous non-small cell lung cancer (NSCLC) remains very difficult to treat, differing remarkably from adenocarcinoma. The knowledge of oncogene molecular markers and their specific targeted therapies have taken place in the management of adenocarcinoma only, forcing patients with squamous disease to settle for several years with convention platinum-based chemotherapy as the best first-line option. The U.S. Food and Drug Administration (FDA), differently from European Agency for Medicine (EMA) in Europe, approved necitumumab combined to first-line regimen of cisplatin plus gemcitabine in squamous patients that however remained an unpopular choice for clinical practice, due to its high side effect profile, not well balanced from limited benefit (1).

Recently, the first-line treatment of advanced NSCLC has been rapidly changed from inhibitors of programmed death receptor 1 (PD-1) and its ligand, with pembrolizumab approval as monotherapy for untreated patients with PD-L1 expression at least $50 \%$ or more, irrespective of histologies (2). This restricted use of first-line pembrolizumab (about 25-30\% of patients) moved research for new therapeutic approaches, that might enhance efficacy of immunotherapy also for those "orphan" patients with PD-L1 lower than 50\%. In 2018, the "practice-changing" phase III Keynote 189 trial crowed the combination of pembrolizumab plus carboplatin/pemetrexed as new standard of care for untreated non-squamous NSCLC, regardless of PD-L1 status (3).

If combining pembrolizumab to chemotherapy should work better also in squamous patients with any PD-L1 levels is recently evaluated in the global phase III KEYNOTE-407 trial (4). A total of 559 untreated patients were randomly assigned to receive four cycle of chemotherapy (carboplatin and paclitaxel or nab-paclitaxel), plus either placebo or pembrolizumab, followed by maintenance of immunoagent or placebo according to patient's initial assignment arm. The trial is specifically devoted to squamous patients, without regard to tumor PD-L1 expression levels. However, patients were stratified according to PD-L1 tumor proportion score (TPS) $<1 \%$ versus $\geq 1 \%$, choice of taxane (paclitaxel versus nab-paclitaxel), and geographic region of enrollment (East Asia versus the rest of world). Notably, more than half of population is PD-L1 positive (63\% of patients) and receive paclitaxel as taxane drug $(60 \%$ of patients). After median follow up of 7.8 months, results of the second interim analysis showed a significant survival improvement in the pembrolizumab-containing arm, with a gain of 4.6 months in terms of median overall survival (OS, HR: 0.64 ) and of 1.6 months in term of progression free survival (PSF, HR: 0.56). The magnitude of survival benefit was similar across all three PD-L1 subgroups, while the reduction on the rate of disease progression was more proportionally linked to PD-L1 expression levels. Higher response rate $(57.9 \%$ vs. $38.4 \%)$ and longer length of response (median duration of response $7.7 \mathrm{vs.} 4.8$ months) were also reported for pembrolizumab combination group, with comparable rates of adverse events between the two treatment groups, except for higher rates of immunorelated toxicities, as expected. These data complement those of non-squamous KEYNOTE-189 trial, but still moving the same questions. First, the predictive role of PD-L1 status fails in both trials of immune-chemo combinations; 
second, the trial design does not clarify whether, in patients with PD-L1 strongly positive tumors, the combination of chemotherapy and pembrolizumab is actually better than pembrolizumab alone, the current therapeutic standard.

Whether the combination of chemotherapy with a different immunoagent - the anti PD-L1 atezolizumabmight represent another potential new first-line treatment option for squamous NSCLC is evaluating in the phase III IMpower 131 trial (5). The largest squamous population was enrolled and included independent of PD-L1 status. Differing from KEYNOTE-407 trial design, patients were randomly assigned to one of three different arms: atezolizumab plus carboplatin/paclitaxel (arm A), atezolizumab plus carboplatin/nab-paclitaxel (arm B), or carboplatin/nab-paclitaxel (arm C, as control arm). Preliminary results, reporting outcomes for arm B and C only, showed that patients assigned to atezolizumab arm experienced an improved PFS relative to those receiving chemotherapy alone, doubling 1-year PFS benefit and reducing the risk of disease progression or death of $29 \%$ (HR PFS: 0.71). The PFS improvement emerged across all PD-L1 expressing subgroups, with greatest benefits for high expression PD-L1 patients, similarly to KEYNOTE-407 trial. However, first interim OS results gave comparable median survivals in overall population (14.0 and 13.9 months for arm B and arm C, respectively). According to PD-L1 status, atezolizumab combined to carboplatin/nab-paclitaxel trended favorably survivals in the high PD-L1 population, improving median OS of 9.5 months (OS, HR: 0.56), but unexpectedly trended worsened survivals in those PDL1 low (OS, HR: 1.34). At European Society of Medical Oncology 2018 congress, the second interim analysis presented by Socinski, confirmed the PFS improvement (PFS, HR: 0.74), as well as comparable median survivals of 14 months in both arms. However, the OS did not cross the boundary and continues to be followed. Notably, findings for the third atezolizumab plus carboplatin/paclitaxel arm are not yet available, as well as the slight separation of survival lines noted by 24 months in intention to treat population-with survival rates of $31.9 \%$ versus $24.1 \%$ for atezolizumab and control arms, respectively-requires a longer follow up to see if an OS difference emerges over time.

Differences in the trial populations (PD-L1 status and post-progression immunotherapy, above all) could explain different results from KEYNOTE-407 and Impower131 trials. Comparing indirectly data, the anti PD-1 pembrolizumab seems to work better than the anti PD-L1 atezolizumab when combined to fist-line chemotherapy in the squamous PD-L1 "all comers" population, reducing the risk of disease progression by $44 \%$, rather than $29 \%$ reported in the atezolizumab trial. Not least, the risk of overtreatment with the immune-chemo combination for PD-L1 strongly positive patients should be also taken into account, considering that pembrolizumab alone reduced risk of death by $65 \%$ in squamous subgroup of KEYNOTE-024 trial (2), more than those reported in the same setting of patients treated with pembrolizumab or atezolizumab plus chemotherapy $(4,5)$.

Finally, the debate on potential biomarkers to select the candidate who will have the best outcomes resultsespecially long survival-from combinations, is far from over. The tumor mutational burden (TMB) has recently emerged as a new predictive biomarker of response to immune checkpoint inhibitors and specifically for the immune combination of nivolumab plus ipilimumab from the largest first-line multipart CheckMate-227 study $(6,7)$. Recently, a FDA/EMA request of data to Bristol-Meyers Squibb regarding survival results for low TMB population of the CheckMate 227 trial, led to a company press-release indicating superimposable survival gain of nivolumab plus ipilimumab as compared to platin-based chemotherapy for high and low TMB groups.

Additional studies follow up and further clinical trials will help to clarify further aspects of the role of combined immune-chemotherapy in advanced squamous NSCLC.

\section{Acknowledgements}

None.

\section{Footnote}

Conflicts of Interest: Dr. Gridelli received honoraria as speaker bureau and advisory board member from Astra Zeneca, BMS, MSD and Roche.

\section{References}

1. Thatcher N, Hirsch FR, Luft AV, et al. Necitumumab plus gemcitabine and cisplatin versus gemcitabine and cisplatin alone as first-line therapy in patients with stage IV squamous non-small-cell lung cancer (SQUIRE): an open-label, randomised, controlled phase 3 trial. Lancet Oncol 2015;16:763-74.

2. Reck M, Rodriguez-Abreu D, Robinson AG, et al. 
Pembrolizumab versus Chemotherapy for PD-L1Positive Non-Small-Cell Lung Cancer. N Engl J Med 2016;375:1823-33.

3. Gandhi L, Rodriguez-Abreu D, Gadgeel S, et al. Pembrolizumab plus Chemotherapy in Metastatic NonSmall-Cell Lung Cancer. N Engl J Med 2018;378:2078-92.

4. Paz-Ares L, Luft A, Vicente D, et al. Pembrolizumab plus chemotherapy for squamous non-small cell lung cancer. $\mathrm{N}$ Engl J Med 2018. [Epub ahead of print].

5. Jotte RM, Cappuzzo F, Vynnychenko I, et al. IMpower131: Primary PFS and safety analysis of a randomized phase III study of atezolizumab + carboplatin + paclitaxel or

Cite this article as: Gridelli C, Casaluce F. The combination strategies will be ready the right first-line choice for squamous lung cancer patients? Transl Lung Cancer Res 2018;7(Suppl 4):S349-S351. doi: 10.21037/tlcr.2018.11.02 nab-paclitaxel vs carboplatin + nab-paclitaxel as $1 \mathrm{~L}$ therapy in advanced squamous NSCLC. J Clin Oncol 2018;36:LBA9000.

6. Hellmann MD, Ciuleanu TE, Pluzanski A, et al. Nivolumab plus Ipilimumab in Lung Cancer with a High Tumor Mutational Burden. N Engl J Med 2018;378:2093-104.

7. Borghaei H, Hellmann MD, Paz-Ares LG, et al. Nivolumab + platinum-doublet chemotherapy vs chemo as firstline treatment for advanced non-small cell lung cancer (NSCLC) with $<1 \%$ tumor PD-L1 expression: Results from CheckMate 227. J Clin Oncol 2018;36:abstr 9001. 